Canadian Science Publishing

Applied Physiology, Nutrition, and Metabolism Physiologie appliquée, nutrition et métabolisme

\title{
The impact of an acute oral phosphate load on endothelium dependent and independent brachial artery vasodilation in healthy males
}

\begin{tabular}{|r|l|}
\hline Journal: & Applied Physiology, Nutrition, and Metabolism \\
\hline Manuscript ID & apnm-2017-0147.R2 \\
\hline Manuscript Type: & Article \\
\hline Date Submitted by the Author: & $17-J u l-2017$ \\
\hline $\begin{array}{r}\text { Complete List of Authors: } \\
\text { Keyword: }\end{array}$ & $\begin{array}{l}\text { Levac, Brendan; Queen's University, School of Kinesiology and Health } \\
\text { Adams, Michael; Queen's University, Biomedical and Molecular Science } \\
\text { Pyke, Kyra; Queen's University, School of Kinesiology and Health Studies }\end{array}$ \\
\hline $\begin{array}{r}\text { Is the invited manuscript for } \\
\text { consideration in a Special } \\
\text { Issue? : }\end{array}$ & $\begin{array}{l}\text { diet, flow-mediated dilation, FMD, vascular smooth muscle cell function, } \\
\text { Phosphorus }\end{array}$ \\
\hline &
\end{tabular}

\section{SCHOLARONE" \\ Manuscripts}


Levac et al., acute oral phosphate load and arterial vasodilation

The impact of an acute oral phosphate load on endothelium dependent and independent brachial artery vasodilation in healthy males

\author{
Brendan M. Levac ${ }^{1}$, Michael A. Adams ${ }^{2}$ and Kyra E. Pyke ${ }^{1}$ \\ ${ }^{1}$ School of Kinesiology and Health Studies, Queen's University, Kingston, Ontario, Canada \\ ${ }^{2}$ Department of Biomedical and Molecular Sciences, Queen's University, Kingston, Ontario, \\ Canada
}

Short Title: acute oral phosphate load and arterial vasodilation

Address Correspondence To:

Dr. Kyra E. Pyke, PhD

Associate Professor

Cardiovascular Stress Response Laboratory

School of Kinesiology and Health Studies

Queen's University

Kingston, ON

K7L 3N6

Tel: (613) 533-6000, x79631

Fax: (613) 533-2009

E-mail: pykek@queensu.ca 


\begin{abstract}
Serum phosphate levels are associated with cardiovascular morbidity and mortality in the general population and endothelial dysfunction may be mechanistically involved. The purpose of this study was to investigate the effects of acute phosphate supplementation on endothelial-dependent (Flow mediated dilation (FMD)) and -independent (Glyceryl trinitrate (GTN)) vasodilation in young, healthy males. 17 healthy male participants ( $23 \pm 3$ years old) were exposed to an oral load of phosphate (PHOS; (liquid supplement containing $1200 \mathrm{mg}$ of phosphorous) and placebo (PLAC) over two experimental days. A brachial artery FMD test was performed pre- and 20 min, $60 \mathrm{~min}$ and $120 \mathrm{~min}$ following the ingestion of the phosphate load or the placebo. GTN tests were performed pre and 140 min post-ingestion. Serum phosphate was not impacted differently by phosphate vs. placebo ingestion $(\mathrm{p}=0.780)$. In contrast, urinary phosphate excretion was markedly increased in the PHOS $(p<0.001)$ but not in the PLAC condition $(p=0.130)(\Delta$ fractional excretion of phosphate in PHOS: 29.2\% vs. PLAC: 9.3\%). This indicates that circulating phosphate levels were homeostatically regulated. GTN-mediated vasodilation was not significantly affected by phosphate ingestion. In primary analysis no impact of phosphate ingestion on FMD was detected. However, when the shear stress stimulus was added as a covariate in a subset of participants, exploratory pairwise comparisons revealed a significantly lower FMD 20min post phosphate ingestion vs. placebo $(p=0.024)$. The effects of phosphate ingestion on FMD and serum phosphate are in contrast with previous findings and the mechanisms that underlie the disparate results require further investigation.
\end{abstract}

Keywords: diet, flow-mediated dilation, FMD, reactive hyperemia, phosphorous, vascular smooth muscle cell function

Abbreviations- AUC- area under the curve, ECG- electrocardiogram, eNOS- endothelial nitric oxide synthase, ET-1 - endothelin-1, FEP- fractional excretion of phosphate, FMD- flow mediated dilation, GTN- Glyceryl trinitrate, HR- heart rate, MAP - mean arterial pressure, NOnitric oxide, PHOS - phosphate condition, PLAC- placebo condition, SS-AUC- Shear stress area under the curve, VSMC - vascular smooth muscle cell 
Levac et al., acute oral phosphate load and arterial vasodilation

\section{Introduction}

Large-scale studies have established that elevated serum phosphate levels are associated with increased atherosclerosis and cardiovascular mortality, even if the magnitude of the rise is not considered to be clinically significant (Dhingra et al. 2007; Onufrak et al. 2008; Tonelli et al. 2005). However, the mechanisms linking changes in serum phosphate to the pathogenesis of vascular disease remain to be established.

The onset of endothelial dysfunction has been described as an early pathological shift away from the pro-vasodilatory, anti-coagulative, and anti-inflammatory phenotype that exerts a protective role in the circulation (Davignon and Ganz 2004). Recent studies, assessing the role of the endothelium in phosphate-induced pathogenesis (Di Marco et al. 2008; Di Marco et al. 2013; Shuto et al. 2009), have demonstrated in vitro that hyperphosphatemia impairs microvascular function, angiogenesis, and endothelial nitric oxide (NO) production and induces endothelial cell apoptosis via increases in reactive oxygen species (Di Marco et al. 2008;Di Marco et al. 2013; Shuto et al. 2009).

Two studies from the same group have assessed endothelial function following a large oral phosphate intake in humans. A single high phosphate meal in young, healthy men raised circulating phosphate levels and resulted in FMD impairment 1-4h post ingestion (Shuto et al. 2009; Nishi et al. 2015). Although thought-provoking, further studies are needed to assess FMD concomitantly with endothelium-independent vasodilation and the magnitude of the shear stress stimulus. These measures are essential in order to determine whether depressed post-phosphate 
FMD relates to a diminished shear stress stimulus or impaired smooth muscle relaxation rather than a dysfunctional endothelium.

With this as context, the aim of the present study was to examine the impact of an acutely administered, oral phosphate load on brachial artery FMD, the shear stress stimulus, and endothelium-independent vasodilation in young, healthy males.

\section{Methods}

\section{Participants}

Seventeen healthy, male volunteers recruited from the Queen's University community (Kingston, Ontario) participated in the study. The absence of cardiovascular and renal disease was confirmed with a medical screening questionnaire. During the initial visit to the laboratory, participants were introduced to the study protocol and screened to ensure that a clear image of the brachial artery, and strong blood velocity signal could be obtained. Blood pressure was assessed using the average of three measurements in a seated position (BpTRU BPM-100, BpTRU Medical Devices, Coquitlam, BC). Hypertensive ( $>140 / 90 \mathrm{mmHg}$ ) and hypotensive $(<90 / 60 \mathrm{mmHg})$ volunteers were excluded. This study was approved by the Health Sciences Research Ethics Board at Queen's University, and participants signed a consent form approved by the same board.

\section{Experimental Design}

Participants completed two experimental visits separated by a period of at least 48 hours and no more than two weeks. The experiment had a within subjects design such that each participant 
completed both conditions: oral phosphate load and placebo; one visit each. The condition order was counterbalanced (i.e. whether the phosphate or placebo condition was performed first alternated between participants as they were recruited, such that half of the participants performed the phosphate condition first) and double-blinded. The full duration of experimental visits occurred between 1200 and 1800 hours to minimize the potential impact of circadian variation on serum phosphate, renal reabsorption and excretion of phosphate.

Endothelial independent (ingestion of glyceryl trinitrate (GTN $400 \mu \mathrm{g})$ ) and endothelial dependent (FMD) brachial artery vasodilation were assessed pre- and post-ingestion of the phosphate load or placebo as shown in Figure 1. Blood and urine samples were collected at the time points indicated (Fig. 1).

\section{Experimental Procedures}

In the 24-hour period prior to the experimental visit, participants were instructed to avoid exercise, alcohol and caffeine. Participants were provided with a standardized low-phosphate breakfast ( $<60 \mathrm{mg}$ phosphorus) to be consumed 6 hours before the experiment, followed by fasting until arrival at the laboratory. The standardized breakfast contained two regular pieces $(\sim 60 \mathrm{~g})$ of commercially prepared white bread (Dempster's Canada, Maple Leaf Foods, Montreal, QC, Canada) and one $~ 50 \mathrm{~g}$ serving of strawberry jam (Smucker's Pure Strawberry Jam, Smucker Food of Canada, Markham, ON, Canada). All experimental visits occurred in a quiet, temperature-controlled room $\left(21 \pm 1^{\circ} \mathrm{C}\right)$. 
Levac et al., acute oral phosphate load and arterial vasodilation

\section{Participant Monitoring}

Heart rate (HR) was measured continuously using three ECG electrodes placed on the upper chest and lower left abdomen. Blood pressure was monitored using an automated blood pressure device (BPM-100, BpTRU Medical Devices, Coquitlam, BC).

\section{Brachial Artery Blood Velocity and Diameter Measurements}

Brachial blood velocity was determined using Doppler ultrasound operating at $4 \mathrm{MHz}$ (Vivid i2 GE Medical Systems, Mississauga, ON, Canada). The Doppler shift frequency spectrum was analyzed via a Multigon 500P TCD spectral analyzer (Multigon Industries, Yonkers, NY, USA), from which the mean blood velocity was determined as a weighted mean of the spectrum of Doppler shift frequencies. The resultant voltage output from the Multigon spectral analyzer was continuously sampled and recorded (PowerLab, AD Instruments, Colorado Springs, CO, USA) for future analysis.

Brachial artery diameter was obtained using 12MHz B-mode ultrasound (Vivid i2 GE Medical Systems). Ultrasound parameters were set to optimize longitudinal B-mode images of the lumen/arterial wall interface. Data was collected using an insonation angle of $68^{\circ}$, and remained constant between trials. All ultrasound images were acquired from the ultrasound device using a VGA to USB frame grabber (VGA2USB-LR, Epiphan Systems Inc., Ottawa, ON) and recorded as a avi video file on an independent computer using commercially available software (Camtasia Studio 7, TechSmith Corporation, Okemos, MI). 
Levac et al., acute oral phosphate load and arterial vasodilation

\section{Phosphate and Placebo}

Depending on the experimental visit, the participant ingested either the phosphate supplement or placebo after a $10 \mathrm{~min}$ rest period (time $0 \mathrm{~min}$ ) which followed the baseline assessment of endothelial-dependent (FMD) and -independent (GTN) vasodilation (Fig.1). The phosphate supplement ((1200 mg of phosphorus) Phoslax Oral Sodium Phosphates, Odan Laboratories, Pointe-Claire, QC, Canada) was mixed with flavoured (Crystal Light, Kraft Food's) water and consumed orally. The placebo was flavoured water alone. Participants were given $125-250 \mathrm{ml}$ of water every hour to maintain hydration throughout the experimental visits.

\section{Brachial Artery Reactive Hyperemia FMD}

All FMD tests were conducted on the left arm. Following a twenty-minute rest period, brachial artery diameter and blood velocity were recorded for one minute of baseline, the final minute of a five minute forearm occlusion (cuff inflation to $250 \mathrm{mmHg}$ ) and for three minutes following the release of vascular occlusion. The cuff was placed on the participant's forearm at the antecubital fossa (distal to the site of brachial artery ultrasound measurement). The participant rested until the next vascular function test (either FMD or GTN, Fig. 1).

\section{Glyceryl Trinitrate-Mediated Vasodilation}

After a ten-minute rest period, arterial diameter and blood velocity measurements were recorded for one minute before a single metered dose of GTN $(400-\mu \mathrm{g})$ was administered sublingually. Continuous brachial artery diameter and blood velocity recordings were obtained for ten minutes following dosing to determine the peak change in arterial diameter and provide 
an index of NO-dependent vascular smooth muscle cell (VSMC) function. The use of sublingual GTN is an established technique for the assessment of endothelium-independent vasodilation (Corretti et al. 2002).

\section{Blood Sampling}

At the time points indicated in Figure 1, venous blood samples were withdrawn via a catheter (BD Nexiva, $20 \mathrm{GA}$ ). Blood samples were centrifuged at $4^{\circ} \mathrm{C}$ for 10 minutes at 2500 RPM and the serum was separated, aliquotted and stored at $-80^{\circ} \mathrm{C}$ for future analyses of phosphate and creatinine. Whole blood viscosity was analyzed from the first blood sample at a shear rate of $225 \mathrm{~s}^{-1}$ at $37^{\circ} \mathrm{C}$ (Brookfield Viscometer DV-II+ Pro, Middleboro, MA, USA).

\section{Urine Sampling}

A mid-stream urine sample was collected from participants when they arrived at the laboratory, and immediately after the last assessment of GTN-mediated vasodilation. Urine samples were centrifuged at $4{ }^{\circ} \mathrm{C}$ for 10 minutes at $2500 \mathrm{RPM}$ and a portion of the sample was stored at $-80^{\circ} \mathrm{C}$ for future analyses of phosphate and creatinine.

\section{Data Analysis}

\section{Brachial Artery Blood Velocity and Heart Rate}

Blood velocity was analyzed offline in 3-second average time bins using data acquisition software (LabChart, AD Instruments), as previously described (28). Baseline heart rate before each FMD test was analyzed as a 1 minute average using the same data acquisition software.

\section{Brachial Artery Diameter}


Vessel diameter was measured offline using automated edge-detection software (Encoder Analysis FMD, Reed Electronics, Perth, WA, Australia) as previously described (Jazuli and Pyke 2011). The same investigator analyzed all images, and was blinded to the experimental condition and trial. Brachial artery diameters were compiled into 3-second time bins for matching with the corresponding 3-second average blood velocities in order to calculate shear stress.

\section{Shear Stress}

Brachial artery shear stress was determined using the following formula: $\tau=4 \cdot \mu \cdot \frac{v}{d}$; where $\tau=$ shear stress, $\mu=$ blood viscosity, $v=$ mean blood velocity, $d=$ brachial artery diameter (Gnasso et al. 2001). Baseline shear stress was quantified as a 1 min average before each FMD test. The shear stress stimulus was quantified as the shear stress area-under-the-curve (AUC) until peak diameter following the release of vascular occlusion. Five participants were not included in the analysis of shear stress AUC due to technical limitations associated with obtaining a clear velocity signal from the brachial artery during the reactive hyperemia. Two included participants were missing viscosity values for their placebo condition visit. The average viscosity in the placebo condition was used to calculate shear stress for these participants.

\section{Flow-mediated Dilation}

Absolute peak change (absolute FMD) and peak percent change (\%FMD) in artery diameter were assessed to characterize the arterial response to increased shear stress. \%FMD was calculated as the percent change in artery diameter from baseline before vascular occlusion to the 
peak 3 second average artery diameter following release of vascular occlusion. Absolute FMD was quantified as the difference between the same peak and baseline diameters. In six of the 136 total FMD tests, FMD was calculated using the diameter from the last minute of vascular occlusion in place of the baseline diameter. In these six scans, a poor baseline scan was obtained. The following formula is used in calculating \%FMD: $\% \mathrm{FMD}=\left(\right.$ diameter $_{\text {peak }}{ }^{-}$

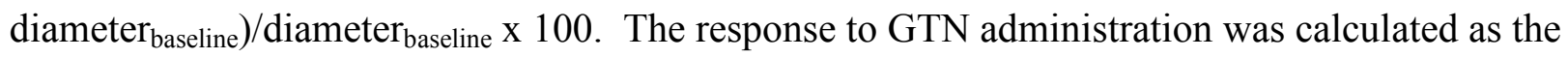
absolute and percent change from the pre GTN baseline to the peak 30 s average in the 10 min post GTN administration.

\section{Phosphate, creatinine, blood lipids and glucose analysis}

Serum and urine phosphate concentrations were analyzed in duplicate using a standard laboratory malachite green phosphate colorimetric assay (Itaya and Ui 1966). Serum and urine creatinine concentrations were analyzed using an enzyme-linked immunosorbent assay (ELISA, Quantichrom Creatinine Assay Kit, Bioassay Systems, Hayward, CA). Duplicate sample coefficient of variation was as follows: serum phosphate $4.3 \%$; urine phosphate $3.1 \%$; serum creatinine $8.2 \%$; urine creatinine $3.7 \%$. The inter-assay $\mathrm{CV}$ (derived from standards run over multiple plates) for the phosphate assay was $5 \%$. The creatinine inter-assay $\mathrm{CV}$ was $3.5 \%$ for urine and 5.7\% for phosphate. All assays were linear $\left(\mathrm{r}^{2} 0.99\right)$. Cholesterol, triglycerides and glucose were analyzed using a Cholestech LDX system from whole blood $(n=14)$. Fractional excretion of phosphate (FEP) was calculated as the ratio of the urinary phosphate excretion rate 
to the renal filtration rate of phosphate according to the following formula (Craver et al. 2013):

$$
F E P=\frac{\text { urinary phosphate } * \text { serum creatinine }}{\text { serum phosphate } * \text { urinary creatinine }} \times 100 \%
$$

Complete blood and urine samples were only obtained successfully in 9 participants due to difficulties with venous access $(n=7)$ or urine analysis $(n=1)$, therefore $n=9$ for FEP analysis.

\section{Statistical Analysis}

All statistical analyses were performed using Sigmaplot 11 or SPSS 24 computer software. All values are expressed, as the mean \pm SD. Statistical significance was set at $\mathrm{p}<0.05$. The variables FMD, GTN mediated vasodilation, shear stress AUC, and serum phosphate levels were compared using a two-way, repeated measures ANOVA. The factors were condition (phosphate (PHOS) vs. placebo (PLAC)) and time (pre-ingestion, and 20, 60 and 120 min postingestion for FMD and pre and 140 min post-ingestion for GTN). FMD data were also analyzed with the shear stress AUC as a covariate (linear mixed model). Anthropometric data were compared between conditions using a paired t-test.

\section{Results}

\section{Participant Characteristics}

Participants were $23 \pm 3$ years old with a BMI of $23.4 \pm 2.7 \mathrm{~kg} / \mathrm{m}^{2}$. Participant characteristics did not differ between the visits to complete the PHOS and PLAC conditions $(\mathrm{p}>0.05)$ (Table 1). The phosphate supplement was well tolerated in all participants.

\section{Mean Arterial Pressure (MAP) and Heart Rate}


MAP (Fig. 2) was not significantly different between the PHOS and PLAC conditions $(\mathrm{p}=0.548)$ and there was no interaction between time and condition $(\mathrm{p}=0.475)$. However, there was a general significant increase over the testing period (main effect of time $\mathrm{p}<0.001$ ). Heart rate also did not differ significantly between conditions $(\mathrm{p}=0.377)$ and there was no interaction between time and condition $(\mathrm{p}=0.597)$. However, there was a modest decrease in heart rate over the testing period such that the pre-ingestion heart rate was higher than all post ingestion heart rates (main effect of time $\mathrm{p}=0.009$; pre-ingestion: $59 \pm 9$ bpm; $20 \mathrm{~min}: 57 \pm 8$ bpm; $60 \mathrm{~min}: 57 \pm$ 8 bpm; 120 min: $57 \pm 7$ bpm).

\section{Serum phosphate concentration}

Although serum phosphate concentrations (Fig. 3A) were significantly greater in the PHOS condition compared to the PLAC condition $(\mathrm{p}=0.030)(\mathrm{n}=10)$, the change from the preingestion baseline was not (Fig 3A insert panel). As a result, there was no time by treatment interaction $(\mathrm{p}=0.780)$. Therefore it appears that the main effect reflects a difference in baseline serum phosphate level between days. There was also an overall significant main effect of time $(\mathrm{p}=0.037)$, with the post hoc analysis identifying that serum phosphate concentration at $60 \mathrm{~min}$ was greater than at $20 \mathrm{~min}(\mathrm{p}=0.05)$. This may reflect some diurnal variation in serum phosphate levels.

\section{Fractional Excretion of Phosphate (FEP)}

Despite the lack of differential impact on serum phosphate, there was a significant interaction between time and condition for FEP ( $p=0.016$; Fig. 3B). The FEP increased 
markedly in the PHOS condition $(\sim 3$ fold; pre vs. post-ingestion $\mathrm{p}<0.001)$. In contrast, in the FEP did not differ pre vs. post-ingestion in the PLAC condition $(p=0.130)$.

\section{Brachial Artery Baseline Diameter}

There was no significant main effect of condition $(\mathrm{p}=0.390)$, or interaction between condition and time $(\mathrm{p}=0.084)$ on brachial artery baseline diameter (Fig. 4). However, there was an overall main effect of time, such that the baseline diameter at 20 and 60 min post phosphate or placebo ingestion was greater than the pre-ingestion baseline diameter $(\mathrm{p}<0.001)$.

\section{Endothelium-independent Vasodilation (GTN)}

There was no main effect of time $(p=0.879)$, or condition $(p=0.132)$, and there was no significant interaction between time and condition for endothelium-independent vasodilation $(p=0.284$; Fig. 5).

\section{Baseline shear stress and Shear Stress Stimulus (SS-AUC)}

The baseline shear stress before the FMD trials did not differ between conditions $(p=0.329)$. However there was a main effect of time such that the pre-ingestion baseline shear rate was higher than in all post-ingestion trials (pre-ingestion $3.9 \pm 2.4 \mathrm{dynes} / \mathrm{cm}^{2} ; 20 \mathrm{~min} 1.7 \pm$ 0.5 dynes $/ \mathrm{cm}^{2} ; 60 \min 1.7 \pm 0.4$ dynes $/ \mathrm{cm}^{2} ; 120 \min 2.2 \pm 1.3$ dynes $/ \mathrm{cm}^{2}$ ). There was no significant interaction between time and condition and no main effect of condition on the SSAUC ( $p=0.444$ and $p=0.064$ respectively) (Fig. 6A). There was a main effect of time $(p<0.001)$ such that the pre-ingestion shear stress stimulus was greater than all other time points $(\mathrm{p}<0.05)$. 


\section{Endothelium-dependent Vasodilation (FMD)}

There was no significant effect of condition or time by condition interaction for \%FMD $(p=0.905$ and $p=0.411$, respectively; Fig. $6 B$ ). Similar to the SS-AUC results, a significant main effect of time on $\%$ FMD was detected $(\mathrm{p}<0.001)$. Although not shown, the pattern of results for absolute FMD were similar to those for \%FMD (Condition $\mathrm{p}=0.952$; time $\mathrm{p}<0.001$; Condition $\mathrm{x}$ Time $\mathrm{p}=0.326)$ ). When FMD was expressed as a change from pre-ingestion, similarly, a main effect of time was detected however there was no significant impact of condition or time by condition interaction (data not shown).

$\%$ FMD was not significantly correlated to serum phosphate concentrations in either the PHOS or PLAC conditions $\left(\mathrm{r}^{2}=0.04, \mathrm{p}=0.228\right.$ and $\mathrm{r}^{2}=0.01, \mathrm{p}=0.508$, respectively).

In the subset of 12 with shear stress AUC data, the pattern of FMD results was the same as for the whole group such that there was a main effect of time, and no effect of condition or condition by time interaction. However, when the shear stress AUC was added to the FMD analysis as a covariate a main effect of condition emerged $(p=0.033)$ (main effect of time $\mathrm{p}=0.007$; time by condition interaction $\mathrm{p}=0.503)$ (Fig. 7). Similar results were found for absFMD: time $p=<0.035$, condition $p=0.041$ and time by condition interaction $p=0.395$. Exploratory comparisons with shear stress AUC as a covariate at each time point revealed a significantly lower percent and absFMD in the PHOS condition at 20 min post ingestion $(p=0.028$ and $p=0.024$; cohen's $d=0.6,33 \%$ lower in PHOS $)$ and a similar trend at 60 min 
$(p=0.051$ and $p=0.063)$. Pre-ingestion FMD and FMD at 120 min post-ingestion were not significantly different between conditions (all $\mathrm{p}>0.5$ ).

\section{Discussion}

The purpose of this study was to determine, in young healthy males, the acute impact of a single oral phosphate load on (i) brachial artery endothelium-independent vasodilation, (ii) the shear stress stimulus elicited with a standard reactive hyperemia protocol, and (iii) the resulting FMD response. The study was performed in young healthy males, with a phosphorous dose of $1200 \mathrm{mg}$, an amount which is 30 percent of the tolerable upper daily intake level (Health Canada 2010). The main findings were that, although there was clear evidence of the bioavailability the phosphate load (i.e. $\sim 3$ fold increase in renal phosphate handling within 2 hours), this amount of phosphate in young healthy males did not significantly: (1) increase serum phosphate, (2) alter VSMC responsiveness to endogenously administered NO, or (3) change the raw FMD response. However, addition of the SS-AUC as a covariate in the FMD analysis revealed a main effect of condition, which was driven by a lower FMD in the phosphate condition at 20 and 60 min postingestion (Fig. 7). These data suggest that there may be a modest, fast acting and transient impact of phosphate ingestion on endothelial function in the absence of a coinciding elevation in serum phosphate.

\section{The Impact of Acute Phosphate Intake on FMD and GTN-mediated Vasodilation}

This study was designed to confirm and extend the findings of the two previous investigations of acute phosphate loading on human endothelial function (Shuto et al. 2009;Nishi 
et al. 2015). Shuto and colleagues (2009) found that in young healthy males there was impaired brachial artery FMD two hours after oral loading with $1200 \mathrm{mg}$ of phosphorous (Pre vs. postprandial FMD: $9.3 \%$ vs. 5.0\%), but not after $400 \mathrm{mg}$ of phosphorous. Furthermore, Shuto et al. (2009) found that the $2 \mathrm{~h}$ postprandial impaired FMD was accompanied by a $20 \%$ increase in serum phosphate. Nishi et al., (2015) extended these findings, again in young healthy males, identifying that meals containing $800 \mathrm{mg}$ and $1200 \mathrm{mg}$ (but not $400 \mathrm{mg}$ ) of phosphorous resulted in FMD impairment 1 through $4 \mathrm{~h}$ postprandial with FMD returning to baseline by the following morning. The magnitude of decline in FMD was associated with the increase in serum phosphate. In contrast, in the present study, serum phosphate was not elevated, and FMD was not impaired $2 \mathrm{~h}$ post $1200 \mathrm{mg}$ supplement ingestion.

Shuto et al., (2009) and Nishi et al., (2015) did not assess VSMC function or the shear stress stimulus for FMD. Therefore it is unclear whether a lower shear stress stimulus or VSMC function contributed to the observations of decreased FMD. In the present study the GTN results suggest that VSMC function was not impacted by phosphate $2 \mathrm{~h}$ post ingestion. A slightly higher pre-supplement SS-AUC persisted at all time points in the PHOS condition, though this did not reach significance $(\mathrm{p}=0.064)$, and the SS-AUC correlated with the FMD responses in both conditions (data not shown). There is no accepted strategy for accounting for variation in the shear stress stimulus when interpreting FMD, although both covariate analysis and ratio normalization have been used (Thijssen et al. 2011). When the SS-AUC was added as a covariate we found a main effect of condition (FMD lower in the phosphate condition) but the interaction 
of time and condition was not significant. Visual inspection of Fig. 7 suggests that the main effect is driven by the $20 \mathrm{~min}$ and $60 \mathrm{~min}$ post ingestion time points. This was supported by additional pairwise comparisons which revealed no differences between conditions at baseline or 120 min post ingestion, while FMD was significantly lower in the phosphate condition at 20 min. Ratio normalization resulted in a somewhat similar trend of results (main effect of condition $p=0.059$; condition comparison at $20 \mathrm{~min} p=0.096$ ) (data not shown). These results from subset analysis are exploratory, however they suggest that our highly bioavailable supplement (see discussion below) was absorbed and handled quickly resulting in a rapid, and transient negative impact on endothelial function. This could happen in the absence of a measured increase in serum phosphate, either due to an increase outside of our sample time points, or absorption and persistence in vascular tissue exposing the endothelium to higher local phosphate concentrations in the absence of an increased serum concentration. However, we cannot rule out that any negative impact on endothelial function was mediated by secondary responses stimulated by ingestion of the phosphate load (e.g. hormonal changes or changes in the concentration of other ions that were not measured).

\section{Factors Influencing Serum Phosphate Changes}

The lack of change in serum phosphate in the present study suggests that at two hours the kidneys had substantively accounted for the oral phosphate load, without serum levels rising; an indication that the phosphate handling mechanisms were very efficient in this cohort of participants. In contrast, in Shuto et al. (2009) and Nishi et al., (2015) the mechanisms of 
phosphate handling were not sufficient such that the $1200 \mathrm{mg}$ (Nishi et al. 2015; Shuto et al. 2009) and 800mg (Nishi et al. 2015) phosphorous loads induced a significant rise in serum phosphate. Thus, differences in the serum phosphate profile between the previous studies (Nishi et al. 2015; Shuto et al. 2009) and the present study support a role for differences in phosphate handling mechanisms (e.g. rate of absorption, changes in the pattern of tissue distribution) being an important determinant of the FMD responses (i.e. the timing and degree of post-ingestion impairment).

In Shuto et al. (2009), the oral phosphate (1200 mg) induced rise in serum phosphate was from moderately high normal to the upper limit of clinically normal ranges. In Nishi et al., ingestion of $800 \mathrm{mg}$ and $1200 \mathrm{mg}$ of phosphorous resulted in increases in serum phosphate to above the normal range. In contrast, the level of serum phosphate observed in this study after loading was similar to the preprandial levels found in Shuto et al., (2009) and Nishi et al., (2015). The lower basal levels in the present trials might have facilitated a greater accommodation capacity for the phosphate load, allowing for rapid and efficient tissue distribution and renal excretion to occur and thereby preventing a significant rise in the serum.

Other differences between the present study and that of Shuto et al. (2009) and Nishi et al., (2015) might also underlie the contrasting results. First, in the present study, an exclusively inorganic phosphate source was administered, whereas Shuto et al. (2009) and Nishi et al., (2015) provided 400mg of the phosphate load as a meal and supplemented the remaining dose in an inorganic form like the present study. Inorganic phosphate salts, such as the liquid oral 
supplement in this study, have a high bioavailability and are readily absorbed (90-100\% vs. 10$60 \%$ of organic sources) by sodium-phosphate co-transporters in the small intestine (Fukagawa et al. 2011; Noori et al. 2010; Virkki et al. 2007). The inorganic phosphate supplement was selected instead of a meal to provide the most bioavailable form of phosphate in an attempt to cause the most rapid and substantial increase in serum phosphate concentrations. However, hyperglycemia or hyperinsulinemia due the meal component in Shuto et al. (2009) and Nishi et al., (2015) may have promoted increased tissue phosphate uptake (Shimamoto et al. 1995;Bergwitz and Juppner 2010), increasing the exposure of the endothelium. Therefore, while the total peak phosphate dose (1200 mg of phosphorus) was consistent between studies, the compositional differences of the ingested phosphate could have affected the absorption and ultimately the exposure of the endothelium via the circulation.

The marked increase in FEP in the PHOS condition in the present study confirms that the phosphate load was largely absorbed, distributed and finally excreted prior to the two hour time point. The ability of the kidney to rapidly promote renal phosphate excretion with increased dietary phosphate is well-documented (Villa-Bellosta et al. 2009;Berndt et al. 2007;Farrow and White 2010;Nishida et al. 2006) and is a likely mechanism, at least in part, for the blunting of the serum phosphate levels observed herein.

Differences in the phenotype of the participants in the present study versus those in Shuto et al. (2009) and Nishi et al., (2015) may have contributed to the different serum phosphate profiles. Participants were recruited from Canada and Japan respectively and there may have 
been national differences in diet (Zhou et al. 2003;Elliott et al. 2008). Chronic exposure to high dietary phosphorus, which may be more likely in a typical Western diet, is a stimulus for downregulating the expression of sodium phosphate co-transporters in the proximal tubule, which acts to decrease phosphate reabsorption (Levi et al. 1994;Portale et al. 1987; Trohler et al. 1976). Therefore, regional variations in chronic dietary phosphate intake may have contributed to enhanced phosphate elimination or tissue distribution in the present study, and generated, at least in part, the disparities in the phosphate disposition. With a higher dose of phosphate $(>1200 \mathrm{mg})$ we may have observed increases in serum phosphate and timing of FMD impairment more similar to Shuto and colleagues (2009) and Nishi and colleagues (2015).

\section{Differences in baseline serum phosphate}

The between-condition difference in baseline serum phosphate concentration in the present study was not expected given the standardized pre-study meal, counterbalanced order of conditions and the within-subjects study design. There are numerous factors that impact serum phosphate concentrations, including diet, time of day, intrarenal mechanisms, age and genetics (Lederer 2014;Kestenbaum et al. 2010). No dietary records were collected for pre-study meals in advance of the breakfast provided, therefore it is possible that participants ate differently in the days preceding each visit.

\section{Interpreting FMD with Variations in Brachial Artery Baseline Diameter}

In the present study, 20 and 60 min post-supplement, brachial artery baseline diameter was significantly greater than pre-supplement (main effect of time, Figure 5). This was an 
Levac et al., acute oral phosphate load and arterial vasodilation

unanticipated outcome of GTN administration, which had a longer lasting vasodilatory effect than expected (Ghiadoni et al. 2000). GTN produces vasodilation by releasing NO, which enters VSMC to activate guanylyl cyclase and increase cGMP production. However, the GTNmediated vasodilation may not be produced entirely through activation of guanylyl cyclase. NO and endothelin-1 (ET-1), a potent vasoconstrictor, coexist in a dynamic balance to regulate vascular tone (Bourque et al. 2011). Specifically, NO plays a fundamental role in modulating ET-1 activity through tonic (Tonelli et al. 2005) inhibition of ET-1 production and/or release, receptor interactions and second messenger signaling (Bourque et al. 2011). In the present study, sustained GTN action, as evidenced by a prolonged increase in brachial artery diameter, may have been induced by persistent NO-mediated inhibition of ET-1 action within the brachial artery even after the metabolism of NO. Conduit artery diameter influences the shear stress stimulus, such that for the same flow, a larger diameter will yield a smaller shear stress. The shear stress stimulus was decreased in both conditions over time, likely influenced by the larger vessel diameter, an effect contributing to the overall lower FMD at 20 minutes. The purpose of this study was to examine the impact of the phosphate load, and this unexpected main effect of time does not diminish the ability to perform that comparison (PHOS vs. PLAC), although it is under conditions of residual GTN dilation at 20 and $60 \mathrm{~min}$.

\section{Baseline MAP and Heart Rate}

MAP increased modestly over time while heart rate was modestly lower in the postingestion vs. pre-ingestion trials. We have observed this pattern of hemodynamic changes with 
rest in the laboratory previously (Pyke and Jazuli 2011). While it is not fully clear why we observed an upward drift in MAP, the absence of an increase in heart rate suggests that it was due to an increase in total vascular resistance rather than an increase in cardiac output. Rest in the cool room $\left(21^{\circ} \mathrm{C}\right)$ may have promoted skin vasoconstriction over time, increasing vascular resistance (Hess et al. 2009; Wilson et al. 2007;Pyke and Jazuli 2011).

\section{Limitations}

In the present study sample, only young, healthy males were recruited. Thus, the impact of phosphate on endothelium-dependent and -independent vasodilation remains to be assessed in females, older adults, and individuals with conditions such as early stages of chronic kidney disease or cardiovascular disease. Serum phosphate concentrations have been shown to be higher in females compared to males (Tonelli et al. 2005), making it important to determine if sex differences in phosphate regulation translate into altered phosphate-related cardiovascular disease risk. In early chronic kidney disease, decreased renal function would impact acute changes in phosphate excretion, thereby increasing the serum phosphate concentrations, enhancing endothelial exposure to phosphate and increasing the likelihood of phosphate induced endothelial dysfunction.

In this study, the serum and urine analyses, over the two hour period, were performed to provide insight into the general pharmacokinetics of the phosphate load (Huang and Moe 2013), thereby confirming that the phosphate dose, at least in part, was absorbed, distributed and excreted (Hruska et al. 2008). However, it was not possible to determine the magnitude of 
Levac et al., acute oral phosphate load and arterial vasodilation

intestinal absorption or tissue distribution from this study. Collecting extensive dietary intake records and multiple 24 hour urine samples may have helped with the interpretation of the interplay between the short and long term effects of dietary phosphate absorption, disposition and excretion.

We were only able to obtain shear stress stimulus data in 12 of our 17 participants, thus our FMD analysis with shear stress as a covariate could only be performed in this subset of 12 . In this analysis there was a main effect of condition, however the time by condition interaction was not significant. Therefore exploratory pairwise comparisons were required to discern that the main effect was driven by condition differences in the post ingestion time points. The interpretation of these findings must include consideration of this subset analysis approach.

\section{Conclusion}

An acute oral phosphate load did not impair FMD or GTN-mediated dilation measured $\sim 2 \mathrm{~h}$ post ingestion. This lack of impact was accompanied by a minimal change in serum phosphate and a marked increase in FEP within 2 hours of phosphate ingestion. The intact FMD and lack of elevation in phosphate at $2 \mathrm{~h}$ is in contrast to two previous investigations (Shuto et al. 2009; Nishi et al. 2015), and may be due to the phenotype of the participants or the nature of the phosphate dose. Exploratory analysis taking into account the magnitude of the shear stress stimulus provided evidence of FMD impairment at 20 and 60 min post supplement ingestion, suggesting a rapid onset transient effect of inorganic phosphate salt ingestion. Further investigations should assess the impact of acute phosphate loads in combination with different 
dietary patterns, meal composition, and in groups that are considered to be at-risk for phosphaterelated cardiovascular disease (e.g. presence of mild renal impairment). Wider scope to the assessments will permit a more comprehensive understanding of the impact of dietary phosphate on vascular function and the role of the mechanisms controlling phosphate disposition, including the kidney, in protecting endothelium-dependent and -independent vasodilation following a high phosphate load.

\section{Acknowledgements}

This study was funded by a Natural Sciences and Engineering Research Council of Canada (NSERC) Discovery Grant and Canada Foundation for Innovation and Ontario Ministry of Research and Innovation leaders Opportunities Funding to K.E. Pyke. B.M. Levac was supported by an NSERC PGS-M scholarship.

\section{Conflict of interest}

The authors have no conflicts of interest to report. 


\section{Reference List}

Bergwitz, C. and Juppner, H. 2010. Regulation of phosphate homeostasis by PTH, vitamin D, and FGF23. Annu. Rev. Med. 61: 91-104.

Berndt, T., Thomas, L. F., Craig, T. A., Sommer, S., Li, X., Bergstralh, E. J.et al. 2007. Evidence for a signaling axis by which intestinal phosphate rapidly modulates renal phosphate reabsorption. Proc. Natl. Acad. Sci. U. S. A. 104(26): 11085-11090.

Bourque, S. L., Davidge, S. T., and Adams, M. A. 2011. The interaction between endothelin-1 and nitric oxide in the vasculature: new perspectives. Am. J. Physiol Regul. Integr. Comp. Physiol. 300(6): R1288-R1295.

Corretti, M. C., Anderson, T. J., Benjamin, E. J., Celermajer, D., Charbonneau, F., Creager, M. A.et al. 2002. Guidelines for the ultrasound assessment of endothelial-dependent flow-mediated vasodilation of the brachial artery: a report of the International Brachial Artery Reactivity Task Force. J. Am. Coll. Cardiol. 39(2): 257-265.

Craver, L., Dusso, A., Martinez-Alonso, M., Sarro, F., Valdivielso, J. M., and Fernandez, E. 2013. A low fractional excretion of Phosphate/Fgf23 ratio is associated with severe abdominal Aortic calcification in stage 3 and 4 kidney disease patients. BMC. Nephrol. 14: 221.

Davignon, J. and Ganz, P. 2004. Role of endothelial dysfunction in atherosclerosis. Circulation, 109(23 Suppl 1): III27-III32.

Dhingra, R., Sullivan, L. M., Fox, C. S., Wang, T. J., D'Agostino, R. B., Sr., Gaziano, J. M.et al. 2007. Relations of serum phosphorus and calcium levels to the incidence of cardiovascular disease in the community. Arch. Intern. Med. 167(9): 879-885.

Di Marco, G. S., Hausberg, M., Hillebrand, U., Rustemeyer, P., Wittkowski, W., Lang, D.et al. 2008. Increased inorganic phosphate induces human endothelial cell apoptosis in vitro. Am. J. Physiol. Renal Physiol. 294(6): F1381-F1387. 
Di Marco, G. S., Konig, M., Stock, C., Wiesinger, A., Hillebrand, U., Reiermann, S.et al. 2013. High phosphate directly affects endothelial function by downregulating annexin II. Kidney Int. 83(2): 213-222.

Elliott, P., Kesteloot, H., Appel, L. J., Dyer, A. R., Ueshima, H., Chan, Q.et al. 2008. Dietary phosphorus and blood pressure: international study of macro- and micro-nutrients and blood pressure. Hypertension, 51(3): 669-675.

Farrow, E. G. and White, K. E. 2010. Recent advances in renal phosphate handling. Nat. Rev. Nephrol. 6(4): 207-217.

Fukagawa, M., Komaba, H., and Miyamoto, K. 2011. Source matters: from phosphorus load to bioavailability. Clin. J. Am. Soc. Nephrol. 6(2): 239-240.

Ghiadoni, L., Donald, A. E., Cropley, M., Mullen, M. J., Oakley, G., Taylor, M.et al. 2000. Mental stress induces transient endothelial dysfunction in humans. Circulation, 102(20): 24732478.

Gnasso, A., Carallo, C., Irace, C., De Franceschi, M. S., Mattioli, P. L., Motti, C.et al. 2001. Association between wall shear stress and flow-mediated vasodilation in healthy men. Atherosclerosis, 156(1): 171-176.

Health Canada. Canadian Dietary Reference Intakes. 2010.

Hess, K. L., Wilson, T. E., Sauder, C. L., Gao, Z., Ray, C. A., and Monahan, K. D. 2009. Aging affects the cardiovascular responses to cold stress in humans. J. Appl. Physiol. 107(4): 10761082.

Hruska, K. A., Mathew, S., Lund, R., Qiu, P., and Pratt, R. 2008. Hyperphosphatemia of chronic kidney disease. Kidney Int. 74(2): 148-157. 
Huang, C. L. and Moe, O. W. 2013. Clinical assessment of phosphorus status, balance and renal handling in normal individuals and in patients with chronic kidney disease. Curr. Opin. Nephrol. Hypertens. 22(4): 452-458.

Itaya, K. and Ui, M. 1966. A new micromethod for the colorimetric determination of inorganic phosphate. Clin. Chim. Acta, 14(3): 361-366.

Jazuli, F. and Pyke, K. E. 2011. The impact of baseline artery diameter on flow-mediated vasodilation: a comparison of brachial and radial artery responses to matched levels of shear stress. Am. J. Physiol. Heart Circ. Physiol. 301(4): H1667-H1677.

Kestenbaum, B., Glazer, N. L., Kottgen, A., Felix, J. F., Hwang, S. J., Liu, Y.et al. 2010. Common genetic variants associate with serum phosphorus concentration. J. Am. Soc. Nephrol. 21(7): 1223-1232.

Lederer, E. 2014. Regulation of serum phosphate. J. Physiol. 592(18): 3985-3995.

Levi, M., Lotscher, M., Sorribas, V., Custer, M., Arar, M., Kaissling, B.et al. 1994. Cellular mechanisms of acute and chronic adaptation of rat renal $\mathrm{P}(\mathrm{i})$ transporter to alterations in dietary P(i). Am. J. Physiol. 267(5 Pt 2): F900-F908.

Nishi, T., Shuto, E., Ogawa, M., Ohya, M., Nakanishi, M., Masuda, M.et al. 2015. Excessive dietary phosphorus intake impairs endothelial function in young healthy men: a time- and dosedependent study. J. Med. Invest. 62(3-4): 167-172.

Nishida, Y., Taketani, Y., Yamanaka-Okumura, H., Imamura, F., Taniguchi, A., Sato, T.et al. 2006. Acute effect of oral phosphate loading on serum fibroblast growth factor 23 levels in healthy men. Kidney Int. 70(12): 2141-2147.

Noori, N., Sims, J. J., Kopple, J. D., Shah, A., Colman, S., Shinaberger, C. S.et al. 2010. Organic and inorganic dietary phosphorus and its management in chronic kidney disease. Iran J. Kidney Dis. 4(2): 89-100. 
Onufrak, S. J., Bellasi, A., Shaw, L. J., Herzog, C. A., Cardarelli, F., Wilson, P. W.et al. 2008. Phosphorus levels are associated with subclinical atherosclerosis in the general population. Atherosclerosis, 199(2): 424-431.

Portale, A. A., Halloran, B. P., and Morris, R. C., Jr. 1987. Dietary intake of phosphorus modulates the circadian rhythm in serum concentration of phosphorus. Implications for the renal production of 1,25-dihydroxyvitamin D. J. Clin. Invest. 80(4): 1147-1154.

Pyke, K. E. and Jazuli, F. 2011. Impact of repeated increases in shear stress via reactive hyperemia and handgrip exercise: no evidence of systematic changes in brachial artery FMD. Am. J. Physiol. Heart Circ. Physiol. 300(3): H1078-H1089.

Shimamoto, K., Higashiura, K., Nakagawa, M., Masuda, A., Shiiki, M., Miyazaki, Y.et al. 1995. Effects of hyperinsulinemia under the euglycemic condition on calcium and phosphate metabolism in non-obese normotensive subjects. Tohoku J. Exp. Med. 177(4): 271-278.

Shuto, E., Taketani, Y., Tanaka, R., Harada, N., Isshiki, M., Sato, M.et al. 2009. Dietary phosphorus acutely impairs endothelial function. J. Am. Soc. Nephrol. 20(7): 1504-1512.

Thijssen, D. H., Black, M. A., Pyke, K. E., Padilla, J., Atkinson, G., Harris, R. A.et al. 2011. Assessment of flow-mediated dilation in humans: a methodological and physiological guideline. Am. J. Physiol. Heart Circ. Physiol. 300(1): H2-12.

Tonelli, M., Sacks, F., Pfeffer, M., Gao, Z., and Curhan, G. 2005. Relation between serum phosphate level and cardiovascular event rate in people with coronary disease. Circulation, 112(17): 2627-2633.

Trohler, U., Bonjour, J. P., and Fleisch, H. 1976. Renal tubular adaptation to dietary phosphorus. Nature, 261(5556): 145-146.

Villa-Bellosta, R., Ravera, S., Sorribas, V., Stange, G., Levi, M., Murer, H.et al. 2009. The Na+Pi cotransporter PiT-2 (SLC20A2) is expressed in the apical membrane of rat renal proximal tubules and regulated by dietary Pi. Am. J. Physiol. Renal Physiol. 296(4): F691-F699. 
Virkki, L. V., Biber, J., Murer, H., and Forster, I. C. 2007. Phosphate transporters: a tale of two solute carrier families. Am. J. Physiol. Renal Physiol. 293(3): F643-F654.

Wilson, T. E., Sauder, C. L., Kearney, M. L., Kuipers, N. T., Leuenberger, U. A., Monahan, K. D.et al. 2007. Skin-surface cooling elicits peripheral and visceral vasoconstriction in humans. J. Appl. Physiol. 103(4): 1257-1262.

Zhou, B. F., Stamler, J., Dennis, B., Moag-Stahlberg, A., Okuda, N., Robertson, C.et al. 2003. Nutrient intakes of middle-aged men and women in China, Japan, United Kingdom, and United States in the late 1990s: the INTERMAP study. J. Hum. Hypertens. 17(9): 623-630. 
Table 1. Participant characteristics

\begin{tabular}{lccc}
\hline & PHOS & PLAC & P value \\
\hline Energy expenditure & $235 \pm 12$ & $238 \pm 15$ & 0.301 \\
$(\mathrm{kcal} / \mathrm{kg} / \mathrm{week})$ & & & \\
Total Cholesterol (mg/dl) & $152.8 \pm 37.7$ & $146.1 \pm 30.7$ & 0.063 \\
Triglycerides (mg/dl) & $86.8 \pm 62.9$ & $73.0 \pm 32.0$ & 0.292 \\
HDL (mg/dl) & $46.2 \pm 14.4$ & $43.9 \pm 10.8$ & 0.205 \\
LDL (mg/dl) & $92.6 \pm 23.7$ & $90.9 \pm 25.8$ & 0.510 \\
Glucose (mg/dl) & $82.9 \pm 10.4$ & $85.1 \pm 5.5$ & 0.402 \\
\hline
\end{tabular}

HDL- high density lipoprotein, LDL- low density lipoprotein. $\mathrm{N}=17$ for energy expenditure and $\mathrm{n}=14$ for blood variables. 


\section{Figure Legends}

Figure 1: Timeline of the protocol. FMD: flow-mediated dilation test, GTN: glyceryl trinitrate test.

Figure 2: MAP recorded before each FMD trial in both the PHOS and PLAC conditions ( $\mathrm{n}=17)$. Times indicate the number of minutes post phosphate or placebo ingestion. MAP increased significantly over the duration of the experiment $(\mathrm{p}<0.001)$. * significantly different from preingestion, ${ }^{\#}$ significantly different from $20 \mathrm{~min},{ }^{\&}$ significantly different from $60 \mathrm{~min}$, PHOS: phosphate condition, PLAC: placebo condition. All data are mean $\pm \mathrm{SD}$.

Figure 3: A- Serum phosphate concentration measured before each FMD trial in both the PHOS and PLAC conditions $(n=10)$. Times indicate the number of minutes post phosphate or placebo ingestion. * PHOS significantly different from PLAC. ${ }^{*} 60$ min significantly different from 20 min. Inset graph illustrates the change in phosphate from pre-ingestion emphasizing the lack of impact of condition on post-ingestion changes. B -Fractional excretion of phosphate (FEP) is presented as the ratio of the urinary phosphate excretion rate to the renal filtration rate of phosphate $(n=9)$. ${ }^{\wedge}$ significantly different from pre-ingestion in PHOS only. PHOS: phosphate condition, PLAC: placebo condition. All data are mean \pm SD.

Figure 4: Brachial artery baseline diameter at the beginning of each FMD trial $(n=17)$. Times indicate the number of minutes post phosphate or placebo ingestion. * significantly different from pre-ingestion, ${ }^{\#}$ significantly different from 60 min post-ingestion, ${ }^{\&}$ significantly different from 120 min post-ingestion. PHOS: phosphate condition, PLAC: placebo condition. All data are mean $\pm \mathrm{SD}$.

Figure 5: Percent change in brachial artery diameter following GTN administration measured pre- and post-phosphate or placebo ingestion $(n=17)$. GTN- Glyceryl trinitrate, PHOS: phosphate condition, PLAC: placebo condition. All data are mean $\pm \mathrm{SD}$.

Figure 6: A- The shear stress stimulus elicited by reactive hyperemia of the forearm, and measured as shear stress area under the curve (SS-AUC) until peak dilation $(n=12)$. B- Percent change in brachial artery diameter $(\% \mathrm{FMD})(\mathrm{n}=17)$. * significantly different from 20 min postingestion, ${ }^{\#}$ significantly different from 60 min post-ingestion, ${ }^{\&}$ significantly different from 120 min post-ingestion. Times indicate the number of minutes post-ingestion. PHOS: phosphate condition, PLAC: placebo condition. All data are mean $\pm \mathrm{SD}$. 
Figure 7: Estimates of \%FMD adjusted for the SS-AUC covariate $(n=12)$. Times indicate the number of minutes post-ingestion. PHOS: phosphate condition, PLAC: placebo condition. All data are mean $\pm \mathrm{SD}$. Although the interaction between condition and time was not significant, exploratory pairwise comparisons at each time point identified a lack of condition differences pre-ingestion and 120 min post ingestion with significant $(p=0.024)$ and near significant $(p=0.051)$ differences at 20 and 60 min post ingestion. * significant difference between PHOS and PLAC. All data are mean \pm SD. 
Figure 1

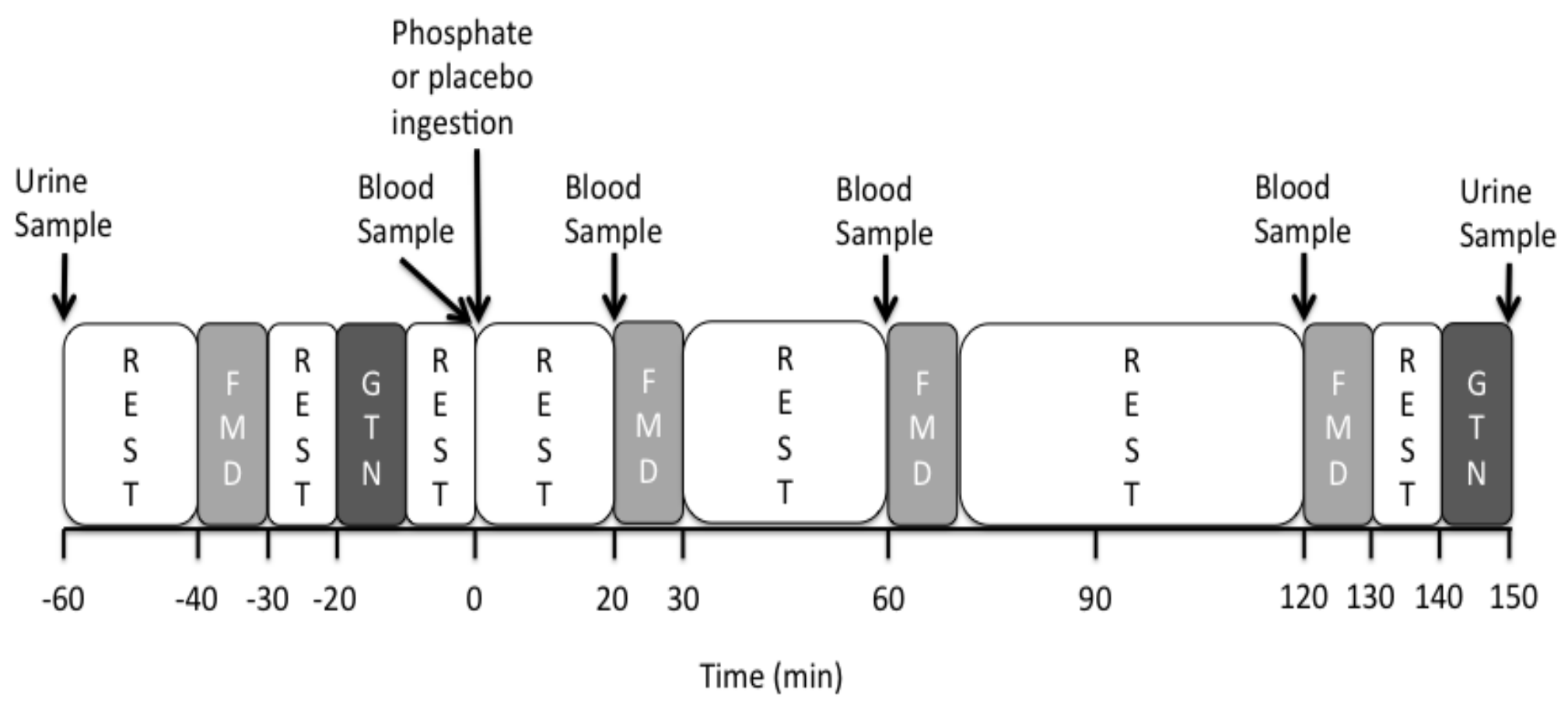

https://mc06.manuscriptcentral.com/apnm-pubs 
Figure 2

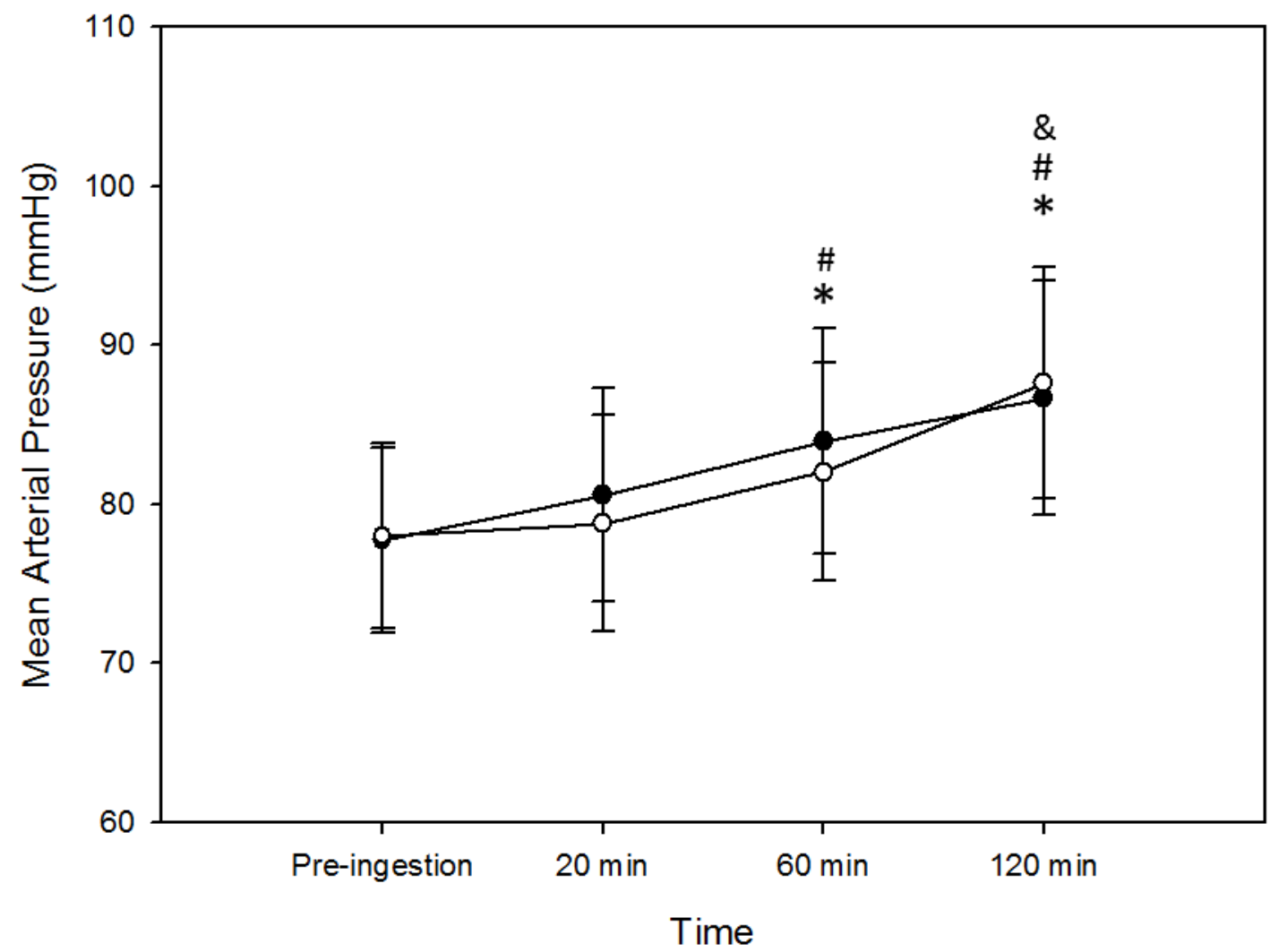

$$
\begin{aligned}
& \longrightarrow \text { PHOS } \\
& \multimap-\text { PLAC }
\end{aligned}
$$

https://mc06.manuscriptcentral.com/apnm-pubs 
Figure 3

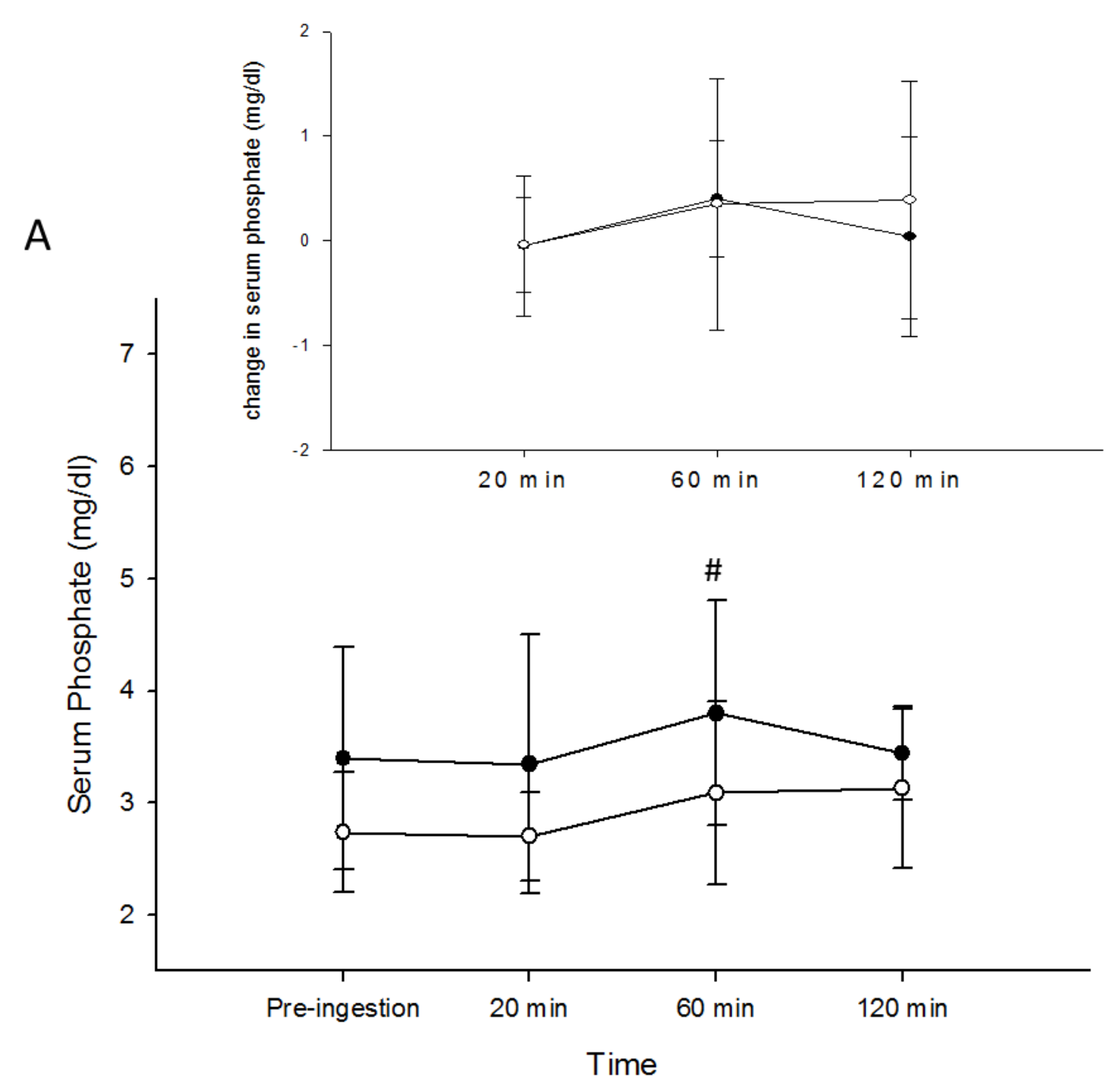

B

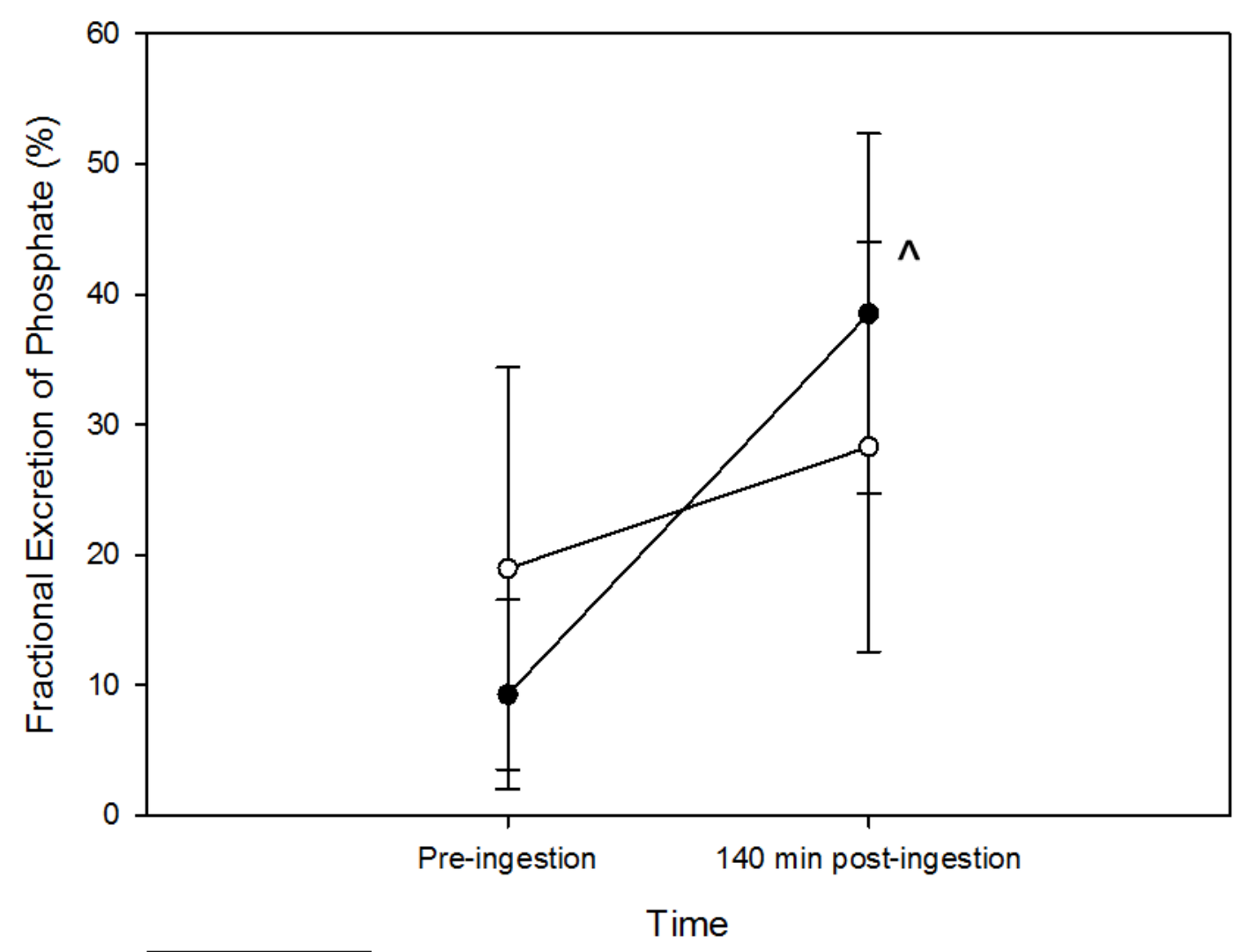

PHOS*

PLAC 


\section{Figure 4}

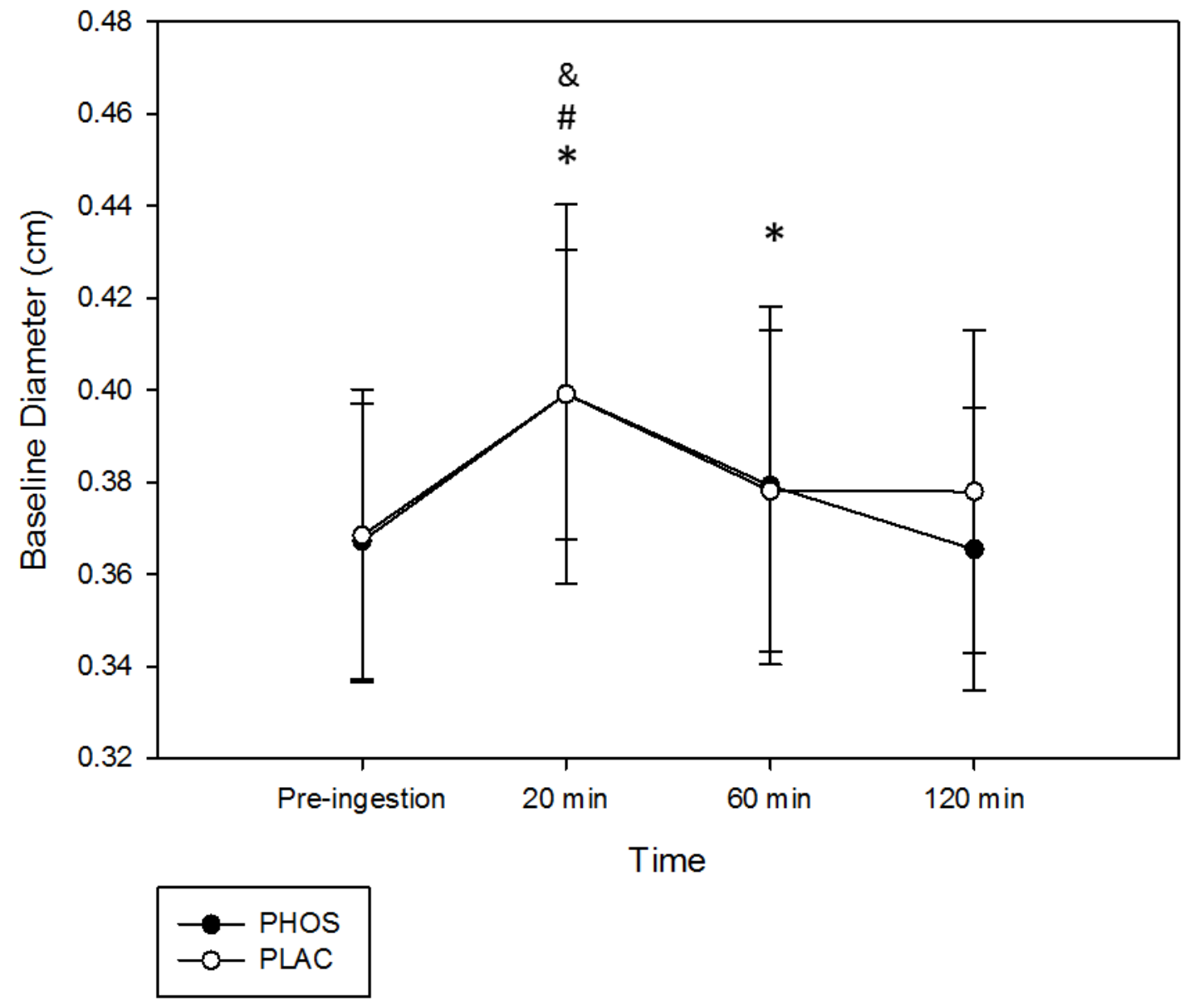

https://mc06.manuscriptcentral.com/apnm-pubs 


\section{Figure 5}

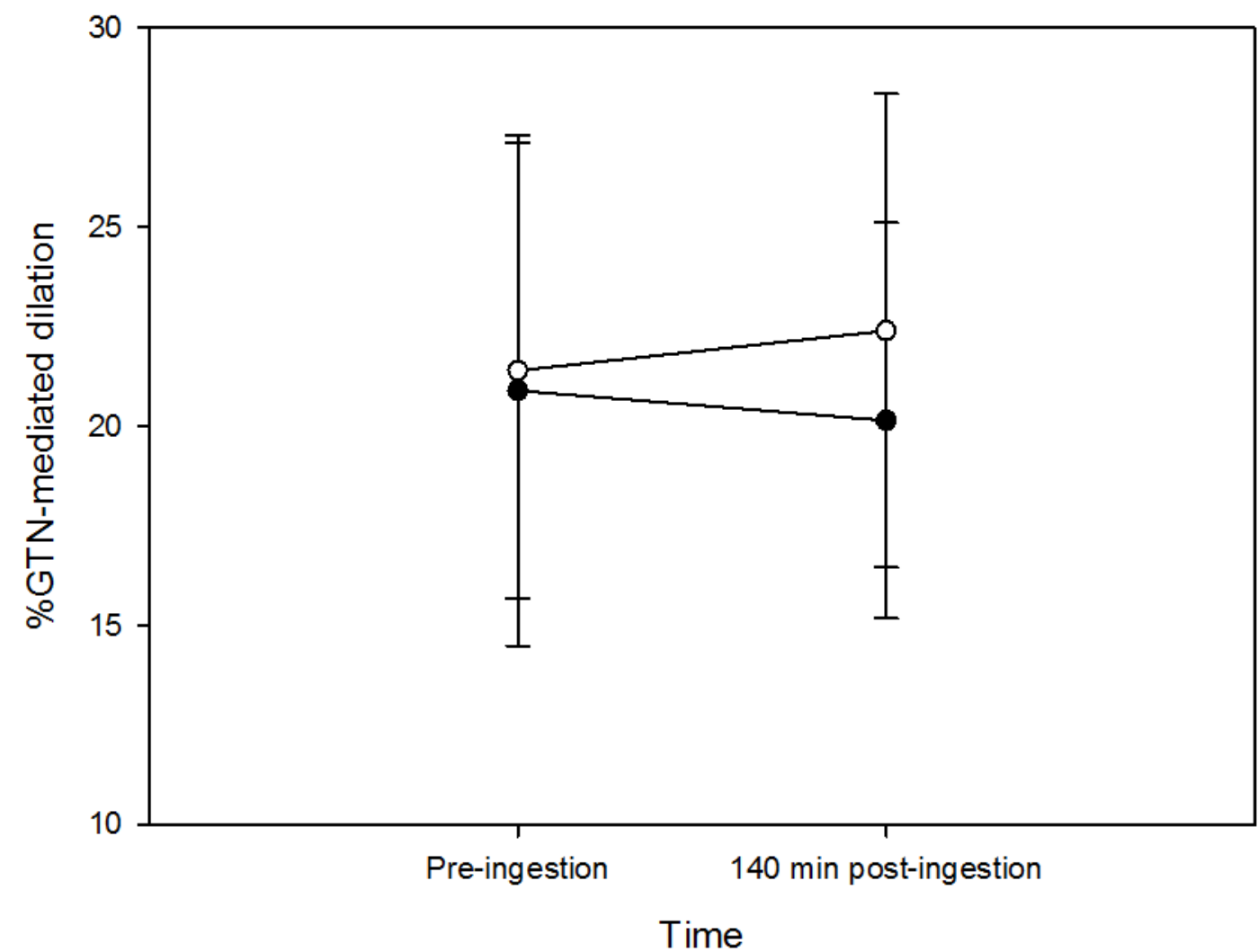

$$
\rightarrow-\text { PHOS }
$$

https://mc06.manuscriptcentral.com/apnm-pubs 
A

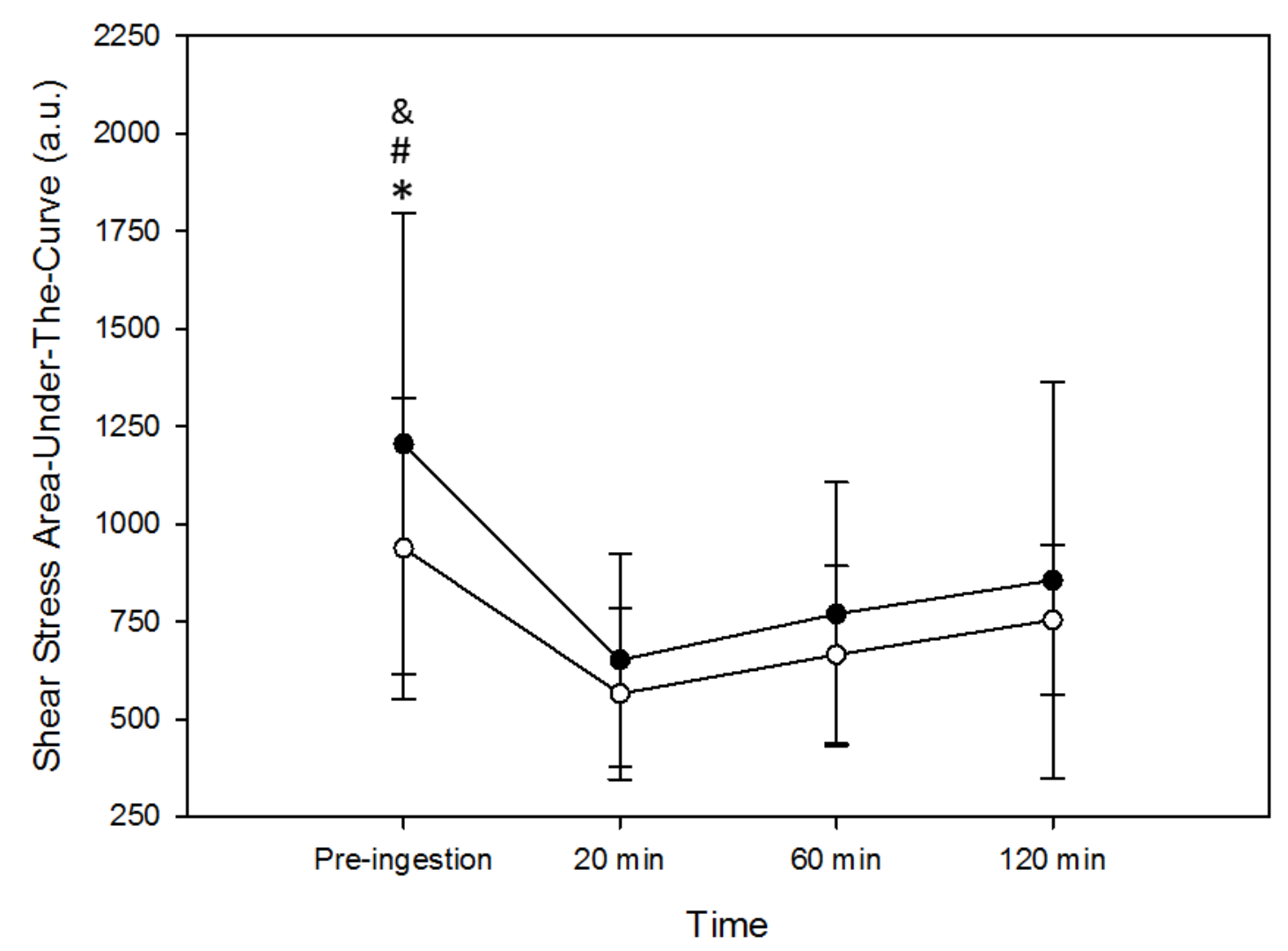

B

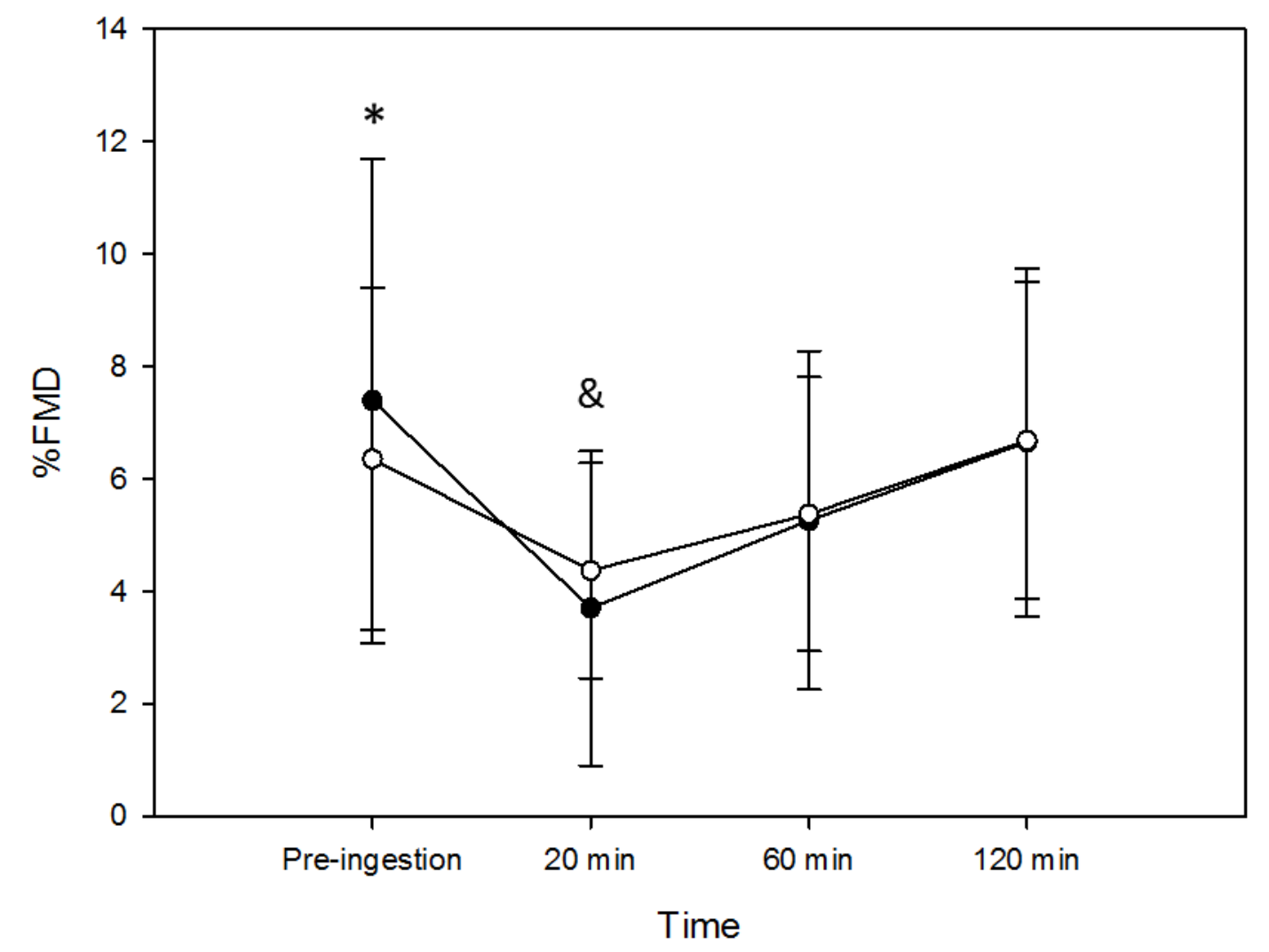




\section{Page 39 of 39}

Applied Physiology, Nutrition, and Metabolism

\section{Figure 7}

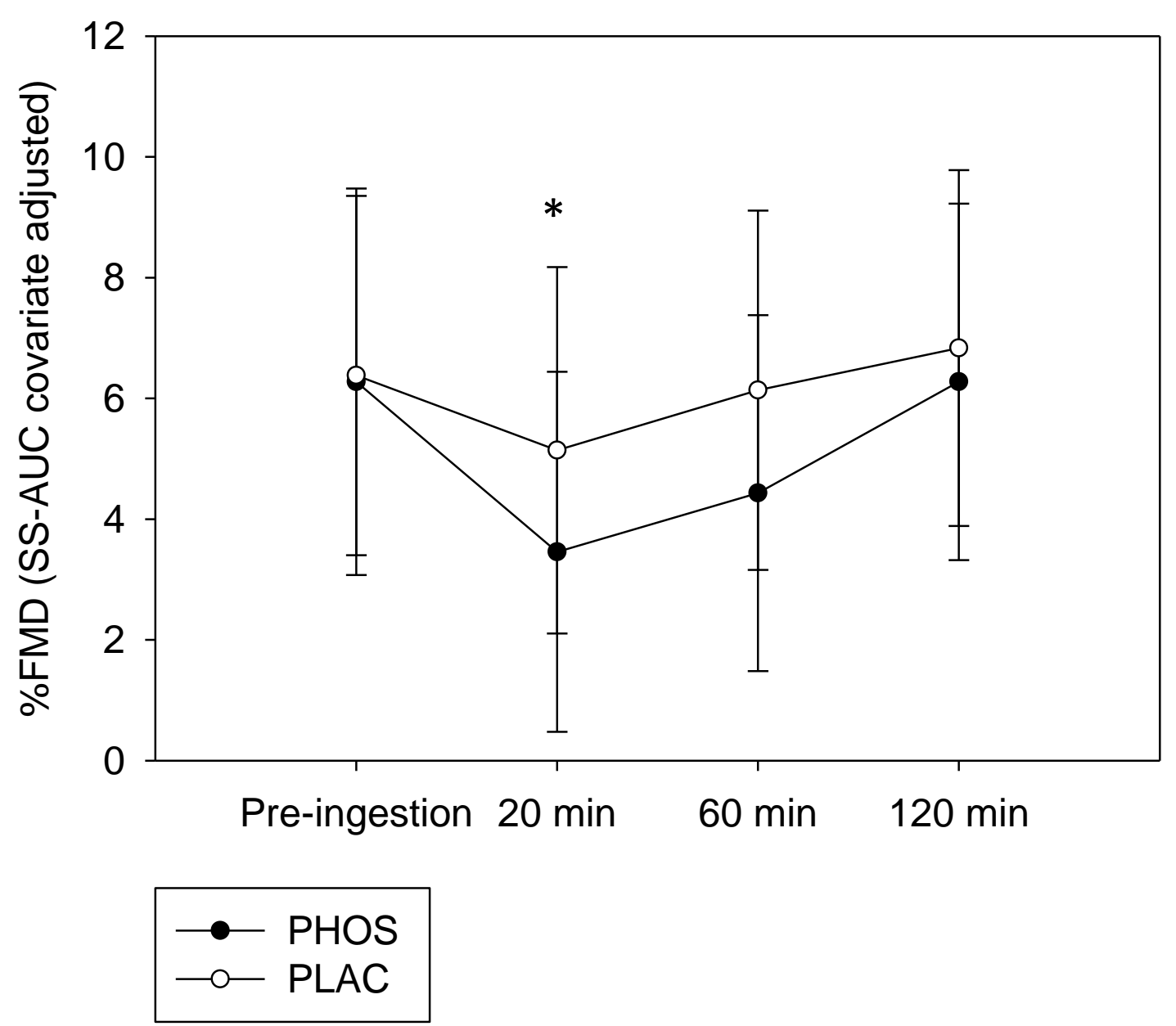

https://mc06.manuscriptcentral.com/apnm-pubs 